\title{
Nonlinear $\mathscr{H}_{\infty}$ almost disturbance decoupling
}

\author{
R. Marino \\ Dipartimento di Ingegneria Elettronica, Università di Rama 'Tor Vergata', Rome, Italy \\ W. Respondek \\ Institute of Mathematics, Polish Academy of Sciences, Warsaw, Poland \\ A.J. van der Schaft \\ Department of Applied Mathematics, University of Twente, Enschede, The Netherlands \\ P. Tomei \\ Istituto di Elettronica, Università di Perugia, Perugia, Italy
}

Received 15 August 1992

Revised 13 September 1993

\begin{abstract}
The $L_{2}$-gain almost disturbance decoupling problem for SISO nonlinear systems is formulated. Sufficient conditions are identified for the existence of a parametrized state feedback controller such that the $L_{2}$-gain from disturbances to output can be made arbitrarily small by increasing its gain. The controller is explicitly constructed using a Lyapunov-based recursive scheme. Sufficient conditions for the solvability of the $\mathscr{H}_{\infty}$ almost disturbance decoupling problem and the explicit construction of the controller are given for a more restrictive class of nonlinear systems.
\end{abstract}

Keywords: Disturbance decoupling; $L_{2}$-gain; high-gain; Lyapunov functions; $\mathscr{H}_{\infty}$ control.

\section{Introduction}

The problem of (exact) disturbance decoupling for nonlinear systems is by now well studied; see the textbooks $[4,12]$ and the references quoted therein. The problem of almost disturbance decoupling for nonlinear systems, as originally introduced for linear systems by Willems [21], however has attracted much less attention. Loosely speaking the aim for this latter problem is to characterize those systems for which disturbance decoupling can be achieved approximately with an arbitrary degree of accuracy. This is rather vague; however it has been shown in $[21]$, see also $[9,16]$, that for linear systems nearly all 'reasonable' mathematical formulations of the almost disturbance decoupling problem lead to the same necessary and sufficient (geometric) conditions. On the other hand, in the nonlinear case one can distinguish between different feasible mathematical formulations of the problem which are not equivalent, and which lead to different problems requiring different mathematical techniques for solving them. In a previous paper [10] we have addressed the nonlinear almost disturbance decoupling problem by using singular perturbation methods, in particular (an infinite-time-interval version of) Tikhonov's theorem. A Lyapunov-based approach to the problem of tracking with arbitrary disturbance attenuation from any initial condition has been recently proposed in [11]. Both treatments of the problem basically consider the induced norm from $L_{\infty}$ 
disturbance functions to $L_{\infty}$ output functions, i.e., for a given $L_{\infty}$-bound on the disturbance function one seeks for a state feedback which, by increasing the gain, attenuates to any desired degree of accuracy the $L_{\infty}$ norm of the difference between the actual output and the output for zero disturbances. On the other hand, it is well known from nonlinear stability theory that also the $L_{2}$-induced norm (or, more classically, the $L_{2}$-gain) is a natural and powerful tool in the analysis of nonlinear systems. Furthermore, the well-known $\mathscr{H}_{\infty}$ optimal control problem for linear systems, although originally formulated in the frequency domain, amounts in its time-domain formulation to the optimal attenuation of the $L_{2}$-induced norm from disturbances to a set of to-be-controlled variables with internal stability. Very recently, cf. $[1,5,19,20]$ it has been shown that this time-domain interpretation of the $\mathscr{H}_{\infty}$ optimal control problem extends naturally to nonlinear systems, and that its solution involves the solution of partial differential equations or inequalities.

Motivated by these developments we consider here the problem of nonlinear almost disturbance decoupling in the $L_{2}$-induced norm from zero initial condition, and call it the nonlinear $L_{2}$-gain almost disturbance decoupling problem. (Recall that for linear systems the almost disturbance decoupling problem is equivalent for every $L_{p}$-induced norm, $p=1,2, \ldots, \infty$, see [21]. Note also that in the linear case there is no loss of generality in restricting to zero initial conditions.) We then impose the additional condition of internal stability and call such a problem nonlinear $\mathscr{H}_{\infty}$ almost disturbance decoupling.

The outline of the paper is as follows. In Section 2 we state and prove the first results (Theorems 2.1 and 2.2) concerning nonlinear $L_{2}$-gain almost disturbance decoupling for single-input single-output nonlinear systems. The sufficient conditions given in Theorem 2.1 are related to those given in [6] for the solution of an adaptive tracking problem. The sufficient conditions for solvability of the problem given in Theorem 2.2 are the same as the ones given in [11], and are thus more general than those of [10]. The proofs do not involve the solution of PDEs: a nonlinear state feedback controller parametrized by a constant 'gain' $k$ is explicitly constructed such that the $L_{2}$-gain is proportional to $1 / k$ and hence can be made arbitrarily small. The approach taken in this paper has the advantage over the one taken in [11] that disturbances are not required to have known $L_{\infty}$-bounds. On the other hand, the result only applies to zero initial conditions, while the results obtained in [11] hold for any initial conditions. We then state, on the basis of Theorem 2.1, a result (Theorem 2.3) concerning the $L_{2}$-gain almost disturbance decoupling with internal stability of the closedloop system ( $\mathscr{H}_{\infty}$ almost disturbance decoupling).

\section{Main results}

We consider the following single-input single-output system:

$$
\begin{aligned}
& \dot{x}=f(x)+g(x) u+\sum_{i=1}^{p} q_{i}(x) \theta_{i}(t), \quad x \in \mathbb{R}^{n}, u \in \mathbb{R}, \\
& y=h(x), \quad y \in \mathbb{R} .
\end{aligned}
$$

In (1) $f: \mathbb{R}^{n} \rightarrow \mathbb{R}^{n}, g: \mathbb{R}^{n} \rightarrow \mathbb{R}^{n}, q_{i}: \mathbb{R}^{n} \rightarrow \mathbb{R}^{n}, h: \mathbb{R}^{n} \rightarrow \mathbb{R}$ are smooth functions, $x$ is the state, $u$ is the input, $\theta_{i}$, $1 \leqslant i \leqslant p$ are the disturbances, whereas $y$ is the output. We assume that $f(0)=0, h(0)=0$.

In this note the following two problems will be addressed.

Definition 2.1. The $L_{2}$-gain almost disturbance decoupling problem is said to be solvable for system (1) if there exists a smooth parametrized state feedback control

$$
u=u(x, k), \quad k \in \mathbb{R}^{+}
$$

such that for every $t, 0 \leqslant t \leqslant T$,

$$
\int_{0}^{t} y^{2}(\tau) \mathrm{d} \tau \leqslant \frac{1}{k} \int_{0}^{t} \theta^{\mathrm{T}}(\tau) \theta(\tau) \mathrm{d} \tau
$$


for the closed-loop system with initial condition $x(0)=0$ and for any disturbance function $\theta(\tau)$ with $[0, T)$ any open interval in which the corresponding solution exists.

Definition 2.2. The $\mathscr{H}_{\infty}$ almost disturbance decoupling problem is said to be solvable for system (1) if the $L_{2}$-gain almost disturbance decoupling problem is solvable by $u=u(x, k), u(0, k)=0, \forall k \in \mathbb{R}^{+}$and the origin is globally asymptotically stable for the closed-loop system with $\theta(t)=0$,

$$
\dot{x}=f(x)+g(x) u(x, k)
$$

Definition 2.3. Let the strong control characteristic index of system (1) be defined as the integer $\rho$ such that

$$
\begin{gathered}
L_{g} L_{f}^{i} h(x)=0, \quad 0 \leqslant i \leqslant \rho-2, \forall x \in \mathbb{R}^{n}, \\
L_{g} L_{f}^{\rho-1} h(x) \neq 0, \quad \forall x \in \mathbb{R}^{n} . \\
\text { If } L_{g} L_{f}^{i} h(x)=0, \forall i, \forall x \in \mathbb{R}^{n} \text {, then } \rho=\infty .
\end{gathered}
$$

Definition 2.4. Let the disturbance characteristic index $v$ of system (1) be defined as the integer such that

$$
\begin{aligned}
& L_{q_{j}} L_{f}^{i} h(x)=0, \quad 1 \leqslant j \leqslant p, 0 \leqslant i \leqslant v-2, \forall x \in \mathbb{R}^{n}, \\
& L_{q_{j}} L_{f}^{v-1} h(x) \neq 0, \quad \text { for some } x \in \mathbb{R}^{n}, \text { some } j, 1 \leqslant j \leqslant p .
\end{aligned}
$$

We assume in the following that $\rho$ is well defined. In this case it is well known $[4,12]$ that the exact disturbance decoupling problem is solvable if and only if $v>\rho$. Hence we will assume throughout in the sequel that $v \leqslant \rho$.

We now give sufficient conditions for the solvability of the $L_{2}$-gain almost disturbance decoupling problem.

Theorem 2.1. If for system (1):

(i) $\rho$ is well defined,

(ii) $\mathscr{G}_{p-1}=\operatorname{span}\left\{g, \operatorname{ad}_{f} g, \ldots, \operatorname{ad}_{f}^{\rho-1} g\right\}$ is involutive and of constant rank $\rho$ in $\mathbb{R}^{n}$,

(iii) $\operatorname{ad}_{q_{i}} \mathscr{G}_{j} \subset \mathscr{G}_{j}, 1 \leqslant i \leqslant p, 0 \leqslant j \leqslant \rho-2$, with $\mathscr{G}_{j}=\operatorname{span}\left\{g, \ldots, \operatorname{ad}_{f}^{j} g\right\}$,

(iv) the vector fields

$$
\tilde{f}=f-\frac{1}{L_{g} L_{f}^{\rho-1} h} L_{f}^{\rho} h, \quad \tilde{g}=\frac{1}{L_{g} L_{f}^{\rho-1} h} g
$$

are complete, then the $L_{2}$-gain almost disturbance decoupling problem is solvable.

Proof. It is well known $([2,4,10,12])$ that under the assumptions (i), (ii) and (iv) we can globally define in $\mathbb{R}^{n}$ a change of coordinates

$$
\begin{gathered}
z_{1}=h(x), \\
\vdots \\
z_{p}=L_{f}^{\rho-1} h(x), \\
z_{\rho+1}=\phi_{\rho+1}(x), \\
\vdots \\
z_{n}=\phi_{n}(x),
\end{gathered}
$$

with $\phi_{i}(x), \rho+1 \leqslant i \leqslant n, \phi_{i}(0)=0$, such that

$$
\left\langle\mathrm{d} \phi_{i}, \mathscr{G}_{\rho-1}\right\rangle=0
$$


and a state feedback

$$
v=L_{g} L_{f}^{p-1} h(x) u+L_{f}^{\rho} h(x)
$$

which globally transform (1) into

$$
\begin{aligned}
& \dot{z}_{1}=z_{2}+\sum_{i=1}^{p} \theta_{i} L_{q_{i}} h(x) \triangleq z_{2}+W_{1}^{\mathrm{T}}(z) \theta, \\
& \quad \vdots \\
& \dot{z}_{\rho-1}=z_{\rho}+\sum_{i=1}^{p} \theta_{i} L_{q_{i}} L_{f}^{\rho-2} h(x) \triangleq z_{\rho}+W_{\rho-1}^{\mathrm{T}}(z) \theta, \\
& \dot{z}_{\rho}=v+\sum_{i=1}^{p} \theta_{i} L_{q_{i}} L_{f}^{\rho-1} h(x) \triangleq v+W_{\rho}^{\mathrm{T}}(z) \theta, \\
& \dot{z}_{r}=\varphi\left(z_{1}, z_{r}\right)+\Psi^{\mathrm{T}}(z) \theta, \\
& y=z_{1},
\end{aligned}
$$

with $z_{r}=\left(z_{\rho+1}, \ldots, z_{n}\right)$ and $\theta=\left(\theta_{1}, \ldots, \theta_{p}\right)$. In $z$-coordinates we have

$$
\mathscr{G}_{j}=\operatorname{span}\left\{\frac{\partial}{\partial z_{\boldsymbol{\rho}-j}}, \ldots, \frac{\partial}{\partial z_{\rho}}\right\}, \quad 0 \leqslant j \leqslant \rho-1
$$

so that (see also [6]) conditions (iii) imply

$$
\begin{aligned}
& W_{i}^{\mathrm{T}}(z)=W_{i}^{\mathrm{T}}\left(z_{1}, \ldots, z_{i}, z_{r}\right), \quad 1 \leqslant i \leqslant \rho, \\
& \Psi^{\mathrm{T}}(z)=\Psi^{\mathrm{T}}\left(z_{1}, z_{r}\right),
\end{aligned}
$$

i.e. (4) becomes

$$
\begin{aligned}
& \dot{z}_{i}=z_{i+1}+W_{i}^{\top}\left(z_{1}, \ldots, z_{i}, z_{r}\right) \theta, \quad 1 \leqslant i \leqslant \rho-1, \\
& \dot{z}_{\rho}=v+W_{\rho}^{\mathrm{T}}\left(z_{1}, \ldots, z_{\rho}, z_{r}\right) \theta \\
& \dot{z}_{r}=\varphi\left(z_{1}, z_{r}\right)+\Psi^{\mathrm{T}}\left(z_{1}, z_{r}\right) \theta .
\end{aligned}
$$

\section{Define}

$$
z_{2}^{*}=-z_{1}-\frac{1}{4} k z_{1}\left(1+W_{1}^{T}\left(z_{1}, z_{r}\right) W_{1}\left(z_{1}, z_{r}\right)\right)
$$

and consider

$$
V_{1}=\frac{1}{2} z_{1}^{2} \text {. }
$$

Its time derivative with $z_{2}=z_{2}^{*}\left(z_{1}, z_{r}, k\right)$ in (5) is given by

$$
\begin{aligned}
\dot{V}_{1} & =-z_{1}^{2}-\frac{1}{4} k z_{1}^{2}\left(1+W_{1}^{\mathrm{T}} W_{1}\right)+z_{1} W_{1}^{\mathrm{T}} \theta \\
& =-z_{1}^{2}-k\left[\frac{1}{4} z_{1}^{2}\left(1+W_{1}^{\mathrm{T}} W_{1}\right)-\frac{z_{1}}{k} W_{1}^{\mathrm{T}} \theta+\frac{\left(W_{1}^{\mathrm{T}} \theta\right)^{2}}{k^{2}\left(1+W_{1}^{\mathrm{T}} W_{1}\right)}\right]+\frac{\left(W_{1}^{\mathrm{T}} \theta\right)^{2}}{k\left(1+W_{1}^{\mathrm{T}} W_{1}\right)} \\
& =-z_{1}^{2}-k\left(\frac{1}{2} z_{1} \sqrt{1+W_{1}^{\mathrm{T}} W_{1}}-\frac{W_{1}^{\mathrm{T}} \theta}{k \sqrt{1+W_{1}^{\mathrm{T}} W_{1}}}\right)^{2}+\frac{\left(W_{1}^{\mathrm{T}} \theta\right)^{2}}{k\left(1+W_{1}^{\mathrm{T}} W_{1}\right)} \\
& \leqslant-z_{1}^{2}+\frac{\left(W_{1}^{\mathrm{T}} \theta\right)^{2}}{k\left(1+W_{1}^{\mathrm{T}} W_{1}\right)} \leqslant-z_{1}^{2}+\frac{1}{k} \frac{\left\|W_{1}\right\|^{2}}{1+\left\|W_{1}\right\|^{2}}\|\theta\|^{2} \\
& \leqslant-z_{1}^{2}+\frac{1}{k}\|\theta\|^{2} .
\end{aligned}
$$


If $\rho=1$, we set $v=z_{2}^{*}\left(z_{1}, z_{r}, k\right)$ and from (7) and (8), when $z(0)=0$, we have, since $V_{1}(0)=0$ and $V_{1}(x) \geqslant 0$,

$$
-\int_{0}^{t} y^{2}(\tau) \mathrm{d} \tau+\frac{1}{k} \int_{0}^{t}\|\theta(\tau)\|^{2} \mathrm{~d} \tau \geqslant V_{1}(x(t))-V_{1}(0) \geqslant 0,
$$

which implies

$$
\int_{0}^{t} y^{2}(\tau) \mathrm{d} \tau \leqslant \frac{1}{k} \int_{0}^{t} \theta^{\mathrm{T}}(\tau) \theta(\tau) \mathrm{d} \tau .
$$

If $\rho>1$, we prove the following claim (see $[3,7,17]$ for similar arguments in the construction of Lyapunov functions for cascade systems).

Claim. Assume that for a given index $i, 1 \leqslant i \leqslant \rho$, for the system

$$
\begin{aligned}
\dot{z}_{1} & =z_{2}+W_{1}^{\mathrm{T}}\left(z_{1}, z_{r}\right) \theta, \\
& \vdots \\
\dot{z}_{i} & =z_{i+1}+W_{i}^{\mathrm{T}}\left(z_{1}, \ldots, z_{i}, z_{r}\right) \theta
\end{aligned}
$$

there exist $i$ functions

$$
z_{j}^{*}=z_{j}^{*}\left(z_{1}, \ldots, z_{j-1}, z_{r}, k\right), \quad z_{j}^{*}(0, \ldots, 0, k)=0, \quad 2 \leqslant j \leqslant i+1,
$$

such that in new coordinates

$$
\tilde{z}_{1}=z_{1}, \quad \tilde{z}_{j}=z_{j}-z_{j}^{*}\left(z_{1}, \ldots, z_{j-1}, z_{r}, k\right), \quad 2 \leqslant j \leqslant i
$$

the function

$$
V_{i}=\frac{1}{2} \sum_{j=1}^{i} \tilde{z}_{j}^{2}
$$

has time derivative, with $z_{i+1}=z_{i+1}^{*}$ in (9), satisfying the inequality for some positive real $c$

$$
\dot{V}_{i} \leqslant-\sum_{j=1}^{i} \tilde{z}_{j}^{2}+\frac{c}{k}\|\theta\|^{2} .
$$

Then, for the system

$$
\begin{aligned}
& \dot{z}_{1}=z_{2}+W_{1}^{\mathrm{T}}\left(z_{1}, z_{r}\right) \theta \\
& \quad \vdots \\
& \dot{z}_{i+1}=z_{i+2}+W_{i+1}^{\mathrm{T}}\left(z_{1}, \ldots, z_{i+1}, z_{r}\right) \theta
\end{aligned}
$$

there exists a function

$$
z_{i+2}^{*}\left(z_{1}, \ldots, z_{i+1}, z_{r}, k\right), z_{i+2}(0, \ldots, 0, k)=0
$$

such that in new coordinates

$$
\tilde{z}_{j}, \quad 1 \leqslant j \leqslant i, \quad \tilde{z}_{i+1}=z_{i+1}-z_{i+1}^{*}\left(z_{1}, \ldots, z_{i}, z_{r}, k\right)
$$

the function

$$
V_{i+1}=\frac{1}{2} \sum_{j=1}^{i+1} \tilde{z}_{j}^{2}
$$


has time derivative, with $z_{i+2}=z_{i+2}^{*}$ in (14), satisfying the inequality

$$
\dot{V}_{i+1} \leqslant-\sum_{j=1}^{i+1} \tilde{z}_{j}^{2}+\frac{c+1}{k}\|\theta\|^{2} .
$$

Proof of the Claim. Consider the function

$$
V_{i+1}=\frac{1}{2} \sum_{j=1}^{i+1} \tilde{z}_{j}^{2} \text {. }
$$

When $z_{i+2}=z_{i+2}^{*}\left(z_{1}, \ldots, z_{i+1}, z_{r}, k\right)$ in system (14), we have by virtue of the assumption in the claim

$$
\begin{aligned}
\dot{V}_{i+1} \leqslant & -\sum_{j=1}^{i} \tilde{z}_{j}^{2}+\frac{c}{k}\|\theta\|^{2} \\
& +\tilde{z}_{i+1}\left[\tilde{z}_{i}+W_{i+1}^{\mathrm{T}} \theta-\sum_{j=1}^{i} \frac{\partial z_{i+1}^{*}}{\partial z_{j}}\left(z_{j+1}+W_{j}^{\mathrm{T}} \theta\right)-\frac{\partial z_{i+1}^{*}}{\partial z_{r}}\left(\varphi+\Psi^{\mathrm{T}} \theta\right)+z_{i+2}^{*}\right] .
\end{aligned}
$$

Defining

$$
\begin{aligned}
& \alpha_{1}\left(z_{1}, \ldots, z_{i+1}, z_{r}\right)=\tilde{z}_{i}-\sum_{j=1}^{i} \frac{\partial z_{i+1}^{*}}{\partial z_{j}} z_{j+1}-\frac{\partial z_{i+1}^{*}}{\partial z_{r}} \varphi, \\
& \alpha_{2}\left(z_{1}, \ldots, z_{i+1}, z_{r}\right)=W_{i+1}-\sum_{j=1}^{i} \frac{\partial z_{i+1}^{*}}{\partial z_{j}} W_{j}-\Psi\left(\frac{\partial z_{i+1}^{*}}{\partial z_{r}}\right)^{\mathrm{T}}, \\
& z_{i+2}^{*}\left(z_{1}, \ldots, z_{i+1}, z_{r}\right)=-\alpha_{1}-\tilde{z}_{i+1}-\frac{1}{4} k \tilde{z}_{i+1}\left(1+\alpha_{2}^{\mathrm{T}} \alpha_{2}\right),
\end{aligned}
$$

equation (19) becomes

$$
\begin{aligned}
\dot{V}_{i+1} & \leqslant-\sum_{j=1}^{i+1} \tilde{z}_{j}^{2}+\tilde{z}_{i+1} \alpha_{2}^{\mathrm{T}} \theta-\frac{1}{4} k \tilde{z}_{i+1}^{2}\left(1+\alpha_{2}^{\mathrm{T}} \alpha_{2}\right)+\frac{c}{k}\|\theta\|^{2} \\
& =-\sum_{j=1}^{i+1} \tilde{z}_{j}^{2}-k\left[\frac{1}{4} \tilde{z}_{i+1}^{2}\left(1+\alpha_{2}^{\mathrm{T}} \alpha_{2}\right)-\frac{\tilde{z}_{i+1}}{k} \alpha_{2}^{\mathrm{T}} \theta+\frac{\left(\alpha_{2}^{\mathrm{T}} \theta\right)^{2}}{k^{2}\left(1+\alpha_{2}^{\mathrm{T}} \alpha_{2}\right)}\right]+\frac{\left(\alpha_{2}^{\mathrm{T}} \theta\right)^{2}}{k\left(1+\alpha_{2}^{\mathrm{T}} \alpha_{2}\right)}+\frac{c}{k}\|\theta\|^{2} \\
& =-\sum_{j=1}^{i+1} \tilde{z}_{j}^{2}-k\left[\frac{1}{2} \tilde{z}_{i+1} \sqrt{1+\alpha_{2}^{\mathrm{T}} \alpha_{2}}-\frac{\alpha_{2}^{\mathrm{T}} \theta}{k \sqrt{1+\alpha_{2}^{\mathrm{T}} \alpha_{2}}}\right]^{2}+\frac{\left(\alpha_{2}^{\mathrm{T}} \theta\right)^{2}}{k\left(1+\alpha_{2}^{\mathrm{T}} \alpha_{2}\right)}+\frac{c}{k}\|\theta\|^{2} \\
& \leqslant-\sum_{j=1}^{i+1} \tilde{z}_{j}^{2}+\frac{c+1}{k}\|\theta\|^{2}
\end{aligned}
$$

which concludes the proof of the claim.

Proof of Theorem 2.1 (Conclusion). Since the assumptions of the claim were shown to hold with $c=1$ for the system

$$
\dot{z}_{1}=z_{2}+W_{1}^{\mathrm{T}}\left(z_{1}, z_{\mathrm{r}}\right) \theta
$$

we apply the claim $(\rho-1)$ times so that a state feedback control

$$
v=z_{\rho+1}^{*}\left(z_{1}, \ldots, z_{\rho}, z_{r}, k\right)
$$

is iteratively built. For the closed-loop system, we have

$$
\dot{V}_{\rho} \leqslant-\sum_{j=1}^{\rho} \tilde{z}_{j}^{2}+\frac{\rho}{k}\|\theta\|^{2}
$$


with

$$
V_{\rho}=\frac{1}{2} \sum_{j=1}^{\rho} \tilde{z}_{j}^{2}
$$

When $z(0)=0$ from (21) and (22), we obtain

$$
-\int_{0}^{t} y^{2}(\tau) \mathrm{d} \tau-\sum_{j=2}^{\rho} \int_{0}^{t} \tilde{z}_{j}^{2}(\tau) \mathrm{d} \tau+\frac{\rho}{k} \int_{0}^{t} \theta^{\mathbf{T}}(\tau) \theta(\tau) \mathrm{d} \tau \geqslant V_{\rho}(x(t))-V_{\rho}(0) \geqslant 0,
$$

which implies, since $V_{\rho}(0)=0$,

$$
\int_{0}^{t} y^{2}(\tau) \mathrm{d} \tau \leqslant \frac{\rho}{k} \int_{0}^{t} \theta^{\mathrm{T}}(\tau) \theta(\tau) \mathrm{d} \tau
$$

Since $k$ can be arbitrarily chosen, we have shown that the $L_{2}$-gain almost disturbance decoupling problem is solvable.

Remark 2.1. For nonzero initial condition $x(0)$ we immediately obtain from (23) the inequality

$$
\int_{0}^{t} y^{2}(\tau) \mathrm{d} \tau \leqslant \frac{\rho}{k} \int_{0}^{t} \theta^{\mathrm{T}}(\tau) \theta(\tau) \mathrm{d} \tau+V_{\rho}(x(0))
$$

with $V_{\rho}$ as given in (22).

Remark 2.2. Conditions (i)-(iii) have been used in [6] as sufficient conditions in order to solve an adaptive tracking problem when $\theta(t)$ are constant parameters (condition (iii) is called strict feedback condition in [6]).

The constructive proof of Theorem 2.1 leads also to a different set of sufficient conditions for the solvability of the $L_{2}$-gain almost disturbance decoupling problem.

Theorem 2.2. If for system (1):

(i) $\rho$ is well defined,

(ii) $\mathrm{d}\left(L_{q_{j}} L_{f}^{i} h\right) \in \operatorname{span}\left\{\mathrm{d} h, \mathrm{~d}\left(L_{f} h\right), \ldots, \mathrm{d}\left(L_{f}^{i} h\right)\right\}, v-1 \leqslant i \leqslant \rho-1,1 \leqslant j \leqslant p, \forall x \in \mathbb{R}^{n}$,

(iii) the vector fields

$$
\tilde{f}=f-\frac{1}{L_{g} L_{f}^{p-1} h} L_{f}^{\rho} h, \quad \tilde{g}=\frac{1}{L_{g} L_{f}^{p-1} h} g
$$

are complete, then the $L_{2}$-gain almost disturbance decoupling problem is solvable.

Proof. Conditions (i)-(iii) allow us to define the global change of coordinates

$$
\begin{gathered}
z_{1}=h(x), \\
\vdots \\
z_{\rho}=L_{f}^{\rho-1} h(x), \\
z_{\rho+1}=\phi_{\rho+1}(x), \\
\quad \vdots \\
z_{n}=\phi_{n}(x),
\end{gathered}
$$


with $\phi_{i}(0)=0,\left\langle\mathrm{~d} \phi_{i}, g\right\rangle=0, \rho+1 \leqslant i \leqslant n$, and the state feedback (3) which globally transform (1) into (see also [18])

$$
\begin{aligned}
& \dot{z}_{i}=z_{i+1}+W_{i}^{\mathrm{T}}\left(z_{1}, \ldots, z_{i}\right) \theta, \quad 1 \leqslant i \leqslant \rho-1, \\
& \dot{z}_{\rho}=v+W_{\rho}^{\mathrm{T}}\left(z_{1}, \ldots, z_{\rho}\right) \theta, \\
& \dot{z}_{r}=\varphi(z)+\Psi^{\mathrm{T}}(z) \theta,
\end{aligned}
$$

with $z_{r}=\left(z_{p+1}, \ldots, z_{n}\right)$. At this point the proof follows the steps of the proof of Theorem 2.1 with (5) replaced by (26).

Remark 2.3. Condition (ii) of Theorem 2.2 requires functions $W_{1}, \ldots, W_{\rho}$ not to depend on $z_{r}$ while conditions (ii), (iii) of Theorem 2.1 require the $z_{r}$-dynamics to be independent of $z_{2}, \ldots, z_{\rho}$.

The next result shows that if in addition to the assumptions required by Theorem 2.1 the zero dynamics (see $[4,12]$ for its coordinate-free definition) are independent of $\theta$, namely

$$
\dot{z}_{r}=\varphi\left(0, z_{r}\right)
$$

and are such that the origin $z_{r}=0$ is globally asymptotically stable, then a solution exists for the problem given in Definition 2.2 .

Theorem 2.3. If, in addition to conditions (i)-(iv) of Theorem 2.1, system (1) is such that (v) the zero dynamics are independent of $\theta$ and globally asymptotically stable, then the $\mathscr{H}_{\infty}$ almost disturbance decoupling problem is solvable.

Proof. We follow the proof of Theorem 2.1 up to (5) which, since the zero dynamics are independent of $\theta$, may be rewritten as

$$
\begin{aligned}
& \dot{z}_{i}=z_{i+1}+W_{i}^{\mathrm{T}}\left(z_{1}, \ldots, z_{i}, z_{r}\right) \theta, \quad 1 \leqslant i \leqslant \rho-1, \\
& \dot{z}_{\rho}=v+W_{\rho}^{\mathrm{T}}\left(z_{1}, \ldots, z_{\rho}, z_{r}\right) \theta \\
& \dot{z}_{r}=\varphi\left(0, z_{r}\right)+z_{1}\left(\varphi_{1}\left(z_{1}, z_{r}\right)+\Psi_{1}^{\mathrm{T}}\left(z_{1}, z_{r}\right) \theta\right)
\end{aligned}
$$

with $\varphi_{1}$ and $\Psi_{1}$ suitable functions. Since the zero dynamics are globally asymptotically stable, by a converse Lyapunov theorem (see [8, p. 31]) there exists a radially unbounded Lyapunov function $V_{0}\left(z_{r}\right)$ such that $\left\langle\mathrm{d} V_{0}, \varphi\left(0, z_{r}\right)\right\rangle$ is negative definite. Consider the function

$$
V_{01}=V_{0}\left(z_{r}\right)+\frac{1}{2} z_{1}^{2} \text {. }
$$

Its time derivative is

$$
\dot{V}_{01}=\left\langle\mathrm{d} V_{0}, \varphi\left(0, z_{r}\right)\right\rangle+z_{1}\left\langle\mathrm{~d} V_{0}, \varphi_{1}\right\rangle+z_{1} z_{2}+z_{1}\left(W_{1}^{\mathrm{T}}+\left\langle\mathrm{d} V_{0}, \Psi_{1}^{\mathrm{T}}\right\rangle\right) \theta
$$

Let

$$
\bar{W}_{1}^{\mathrm{T}}\left(z_{1}, z_{r}\right)=W_{1}^{\mathrm{T}}\left(z_{1}, z_{r}\right)+\left\langle\mathrm{d} V_{0}\left(z_{r}\right), \Psi_{1}^{\mathrm{T}}\left(z_{1}, z_{r}\right)\right\rangle
$$

and define

$$
z_{02}^{*}\left(z_{1}, z_{r}\right)=-\left\langle\mathrm{d} V_{0}, \varphi_{1}\right\rangle-z_{1}-\frac{1}{4} k z_{1}\left(1+\bar{W}_{1}^{\mathrm{T}} \bar{W}_{1}\right)
$$

which when substituted in (27) gives (recall the proof of Theorem 2.1)

$$
\dot{V}_{01} \leqslant-z_{1}^{2}+\frac{1}{k}\|\theta\|^{2}+\left\langle\mathrm{d} V_{0}, \varphi\left(0, z_{r}\right)\right\rangle .
$$


Since $\left\langle\mathrm{d} V_{0}, \varphi\left(0, z_{r}\right)\right\rangle$ is negative definite, this implies that

$$
\dot{V}_{01} \leqslant-z_{1}^{2}+\frac{1}{k}\|\theta\|^{2} .
$$

Following the argument used in Theorem 2.1 the $L_{2}$-gain almost disturbance decoupling problem is solved for systems with $\rho=1$. Moreover, from (28) it follows global asymptotic stability of the origin when $\theta=0$. Therefore, when $\rho=1$ the control $v=z_{02}^{*}\left(z_{1}, z_{r}\right)$ solves the $\mathscr{H}_{\infty}$ almost disturbance decoupling problem. The proof for $\rho>1$ is a straightforward extension of the proof given in Theorem 2.1 .

Finally, the connection with nonlinear $\mathscr{H}_{\infty}$ control $[1,5,19,20]$ is as follows. Consider a nonlinear system

$$
\begin{aligned}
& \dot{x}=f(x, u)+q(x) \theta, \quad f(0,0)=0, \\
& z=h(x, u), \quad h(0,0)=0 .
\end{aligned}
$$

The state feedback $\mathscr{H}_{\infty}$ optimal control problem consists in finding the constant $\gamma^{*} \geqslant 0$ having the property that for every $\gamma>\gamma^{*}$ we can construct a state feedback $u=\alpha_{\gamma}(x), \alpha_{\gamma}(0)=0$, such that the closed-loop system is internally stable and the $L_{2}$-gain from $\theta$ to $z$ becomes $\leqslant \gamma$, i.e.

$$
\int_{0}^{t} z^{\mathrm{T}}(\tau) z(\tau) \mathrm{d} \tau \leqslant \gamma^{2} \int_{0}^{t} \theta^{\mathrm{T}}(\tau) \theta(\tau) \mathrm{d} \tau
$$

for all $t \geqslant 0$ and $\theta \in L_{2}(0, t)$, and where $z(\tau)$ is the zero-state response. The problem can be called regular if $\partial h / \partial u(x, u)$ has full column-rank; otherwise it is called singular. It is well known that the singular $\mathscr{H}_{\infty}$ control problem is intrinsically more difficult than the regular problem, see e.g. [13-15] for the linear case. In the present situation we have $z(=y)=h(x)$ and thus the $\mathscr{H}_{\infty}$ optimal control problem is totally singular, while solvability of the $\mathscr{H}_{\infty}$ almost disturbance decoupling problem amounts to the condition $\gamma^{*}=0$; see $[15,16]$ for the linear case.

\section{References}

[1] J.A. Ball, J.W. Helton and M.A. Walker, $\mathscr{H}_{\infty}$ control for nonlinear systems with output feedback, IEEE Trans. Automat. Control 38 (1993) 546-559.

[2] C.I. Byrnes and A. Isidori, Global feedback stabilization of nonlinear systems, in: Proc. IEEE 24th Conf. on Decision and Control, Ft. Lauderdale (1985) 1031-1037.

[3] C.I. Byrnes and A. Isidori, New results and examples in nonlinear feedback stabilization, Systems Control Lett. 12 (1989) 437-442.

[4] A. Isidori, Nonlinear Control Systems (Springer, Berlin, 2nd ed., 1989).

[5] A. Isidori and A. Astolfi, Disturbance attenuation and $\mathscr{H}^{\infty}$-control via measurement feedback in nonlinear systems, IEEE Trans. Automat. Control 37 (1992) 1283-1293.

[6] I. Kanellakopoulos, P.V. Kokotovic and A.S. Morse, Systematic design of adaptive controllers for feedback linearizable systems, IEEE Trans. Automat. Control 36 (1991) 1241-1253.

[7] P.V. Kokotovic and H.J. Sussmann, A positive real condition for global stabilization of nonlinear systems, Systems Control Lett. 12 (1989) 125-134.

[8] N.N. Krasovskii, Stability of Motion (Stanford Univ. Press, Stanford, CA, 1963).

[9] R. Marino, W. Respondek and A.J. van der Schaft, A direct approach to almost disturbance and almost input - output decoupling, Internat. J. Control 48 (1988) 353-383.

[10] R. Marino, W. Respondek and A.J. van der Schaft, Almost disturbance decoupling for single-input single-output nonlinear systems, IEEE Trans. Automat. Control 34 (1989) 1013-1017.

[11] R. Marino, W. Respondek, A.J. van der Schaft and P. Tomei, Tracking control of nonlinear systems with disturbance attenuation, in: Proc. IEEE 31 st Conf. on Decision and Control, Tucson (1992) 2469-2474.

[12] H. Nijmeijer and A.J. van der Schaft, Nonlinear Dynamical Control Systems (Springer, New York, 1990).

[13] C. Scherer, The Riccati inequality and state-space $\mathscr{H}_{\infty}$-optimal control, Ph.D. Thesis, Univ. of Würzburg (1990).

[14] A.A. Stoorvogel, The singular $\mathscr{H}_{\infty}$ control problem with dynamic measurement feedback, SIAM J. Control Optim. 29 (1991) $160-184$.

[15] A.A. Stoorvogel and H.L. Trentelman, The singular $\mathscr{H}_{\infty}$ control problem with state-feedback, SIAM J. Control Optim. 28 (1990) 1190-1208. 
[16] H.L. Trentelman, Almost invariant subspaces and high-gain feedback, CWI Tract No. 29, Amsterdam, The Netherlands, 1986.

[17] J. Tsinias, Sufficient Lyapunov-like conditions for stabilization, Math. Control Signals and Systems 2 (1989) $343-357$.

[18] A.J. van der Schaft, Controlled invariance for Hamiltonian systems, Math. Systems Theory 18 (1985) $257-291$.

[19] A.J. van der Schaft, On a state space approach to nonlinear $\mathscr{H}_{\infty}$ control, Systems Control Lett. 16 (1991) 1-8.

[20] A.J. van der Schaft, $L_{2}$-gain analysis of nonlinear systems and nonlinear state feedback $\mathscr{H}_{\infty}$ control, IEEE Trans. Automat. Control 37 (1992) 770-784.

[21] J.C. Willems, Almost invariant subspaces: an approach to high-gain feedback design - Part I: Almost controlled invariant subspaces, IEEE Trans. Automat. Control 26 (1981) 235-252. 